\title{
Advanced non-small cell lung cancer: the role of PD-L1 inhibitors
}

\author{
David F. Heigener ${ }^{1,2}$, Martin Reck $^{1,3}$ \\ ${ }^{1}$ Lungenclinic Grosshansdorf, Airway Research Center North, German Center for Lung Research, Grosshansdorf, Germany; ${ }^{2}$ Chrisitan-Albrechts- \\ University, Kiel, Germany; ${ }^{3}$ Luebeck University, Luebeck, Germany \\ Contributions: (I) Conception and design: All authors; (II) Administrative support: None; (III) Provision of study materials or patients: None; (IV) \\ Collection and assembly of data: All authors; (V) Data analysis and interpretation: All authors; (VI) Manuscript writing: All authors; (VII) Final \\ approval of manuscript: All authors. \\ Correspondence to: Dr. David F. Heigener, MD, PhD. LungenClinic Grosshansdorf, Woehrendamm 80, 22927 Grosshansdorf, Germany. \\ Email: D.heigener@lungenclinic.de.
}

\begin{abstract}
PD-L1 and PD-1 inhibitors were both developed to combat a huge array of cancers. Both classes of agents block the PD-1/PD-L1 pathway. Unlike PD-1 inhibitors, PD-L1 inhibitors do also block the B-7.1-receptor and leave the PD-L2/PD-1 axis unaffected. Whether these differences enhance efficacy and tolerability is not clear yet. There are three PD-L1 inhibitors approved or in late clinical development: Atezolizumab, approved in $2^{\text {nd }}$-line treatment of non-small cell lung cancer, durvalumab, showing promising results as a consolidation therapy in stage III disease and avelumab, the only drug exploiting antigendependent cytotoxicity. Future directions are the combination of these compounds with chemotherapy or other immuno-oncologic drugs.
\end{abstract}

Keywords: PD-L1 inhibition; atezolizumab; durvalumab; avelumab; non-small cell lung cancer (NSCLC)

Submitted Dec 12, 2017. Accepted for publication Jan 14, 2018.

doi: $10.21037 /$ jtd.2018.01.112

View this article at: http://dx.doi.org/10.21037/jtd.2018.01.112

\section{Introduction}

In 2012, phase I results of the antitumor efficacy in solid tumors of the programmed death receptor 1 (PD-1) antagonistic antibody BMS-936558 and the antibody against its corresponding ligand (PD-L1), BMS-936559 were published "back to back" in the New England Journal of Medicine $(1,2)$. Both showed promising antitumour activity, however, due to slightly better results, the sponsor decided to pursue the development of the PD-1 inhibitor only. Albeit Nivolumab (the drug name of BMS936558) was a tremendous success, gaining approval for the treatment of many cancers, the question arises, whether it was the right decision to abandon the PD-L1 antibody.

Meanwhile, we have phase III clinical data of the PDL1 antibodies Atezolizumab and Durvalumab which lead to approval in the former for second-line treatment of metastatic non-small cell lung cancer (NSCLC) and made the latter the first promising approach as consolidation therapy in stage III NSCLC.
In this review we will focus on biological differences between the two targets and discuss the major clinical results of the PD-L1 antibodies in advanced development.

\section{Key differences between PD-1 and PD-L1 blockade}

PD-L1 and PD-L2 are both ligands competing for the PD-1 receptor with comparable affinities but different kinetics of association and dissociation (3). The binding of PD-L2 to PD-1 can have opposite effects compared to the PD-L1-PD-1 binding when expressed on dendritic cells. Whereas PD-L1 attenuates immune response, binding of PD-L2 results (besides competitive inhibition of PD-L1 binding) in increased expression of CD3 and Inducible CoStimulator (ICOS) on T-cells. This results in enhanced immune response and lower parasitemia in a malariainfected cell model (4). PD-1 Inhibitors potentially inhibit PD-L2 mediated immune enhancement in this setting, whereas PD-L1 inhibitors do not affect it. However, the role of this phenomenon in cancer patients remains unclear. 
Table 1 Description of immunoscore used for atezolizumab trials (Fehrenbacher et al.; Lancet 2016)

\begin{tabular}{lc}
\hline Score & Percentage of PD-L1 expressing cells \\
\hline Tumor cells & $<1 \%$ \\
TC 0 & $\geq 1 \%$ to $<5 \%$ \\
TC 1 & $\geq 5 \%$ to $<50 \%$ \\
TC 2 & $\geq 50 \%$ \\
TC 3 & $<1 \%$ \\
Tumor infiltrating immune cells & $\geq 1 \%$ to $<5 \%$ \\
IC 0 & $\geq 5 \%$ to $<10 \%$ \\
IC 1 & $\geq 10 \%$ \\
IC 2 & \\
IC 3 & \\
\hline
\end{tabular}

In an animal model, PD-L2 attenuates cytokine release from invariant nature killer T-cells (iNKT-cells) and reduced airway hyperreactivity in a murine model of asthma (5). PD-L2 thus seems to play a role in inflammation homeostasis of the lung. Again, PD-L1 Inhibitors do not affect this pathway whereas PD-1 inhibitors could potentially do. Probably, this could lead to lower rates of pneumonitis under PD-L1 compared to PD-1 inhibitors.

A hint for less immunogenic pulmonary toxicity by PDL1 compared to PD-1 inhibitors came from a meta-analysis comparing safety and efficacy of these two antibodies: 3,284 patients treated with PD-1 inhibitors and 2,460 patients treated with PD-L1 inhibitors from 23 studies were analyzed. There was no significant difference in response rate (RR) or overall incidence of adverse events. However, there was a slight increase in immune-related adverse events under PD-1 inhibitors with $16 \%$ compared to $11 \%$ with $\mathrm{PD}-\mathrm{L} 1$ inhibitors $(\mathrm{P}=0.07)$, as well as pneumonitis (4\% under PD-1 vs. $2 \%$ under PD-L1; $\mathrm{P}=0.01$ ). Whether this is due to the above mentioned differential biological mechanisms or just a bias, remains unclear. PD-L1 trials were more often earlier phase trials, so the difference can for example also be due to underreporting when inclusion criteria were not available from all PD-L1 trials due to the lack of a full publication (6).

Another target of PD-L1 is a receptor called B 7.1. (also known as CD80). The interaction between PD-L1 and B 7.1 inhibits T-cell activation and cytokine production (7). By blocking this axis, T-cells can exploit their immunestimulatory effect.
Although, there are some indirect clues that PD-L1 inhibition might be more efficacious and safer than PD-1 inhibition. It is, however, not clear, whether there are clinically relevant differences between these two types of antibodies in these terms.

\section{Clinical data}

\section{Atezolizumab}

Atezolizumab (MPDL-3280A; Tecentriq $^{\circ}$ ) is an IgG1-Type monoclonal antibody lacking antigen-dependent cytotoxicity (ADCC). To date it is the only approved PD-L 1 directed antibody for the treatment of NSCLC in Europe. In the phase III OAK trial patients with NSCLC who had received one or two prior lines of therapy were randomized to receive either Atezolizumab (1,200 mg fixed dose every 3 weeks) or Docetaxel in a 1:1 proportion. Co-primary endpoints were progression-free survival (PFS) and overall survival (OS). For the primary analysis, 425 patients in either arm were sufficient (the remainder was necessary to power subsequent analyses). About $75 \%$ of patients entered the study for second line, and $25 \%$ for third-line treatment. OS in the intentto-treat population was 13.8 months under Atezolizumab compared to 9.6 months under docetaxel [hazard ratio $(\mathrm{HR})=0.73, \mathrm{P}=0.0003]$. There was no significant difference regarding histologic subtype (i.e., squamous versus nonsquamous). The only subgroup which does not seem to benefit from Checkpoint inhibition is the group of patients with activating driver mutations in the epidermal-growth factor receptor (EGFR) gene or with a rearrangement between the echinoderm-microtubule associated ligand-4 and anaplastic lymphoma kinase genes (EML4-ALK). This was underscored by a meta-analysis from second-line trials with PD-1 inhibitors (8). Notably the PFS in the ITTGroup was similar in both arms, only showing superiority for atezolizumab in the patients with high PD-L1 expression in the tumor (HR $=0.63, \mathrm{P}$ value not reported) (9) (Table 1). The detailed results are shown in Table 2.

Of note, fewer side effects occurred under atezolizumab compared with chemotherapy. Severe side effects occurred in $15 \%$ and $43 \%$, respectively.

In the phase III IMpower 150 trial, patients with previously untreated stage IV non-squamous NSCLC received carboplatin, paclitaxel, bevacizumab with or without atezolizumab as first-line regimen, a third arm, carboplatin, paclitaxel and atezolizumab, was not reported in this preliminary publication. The median PFS was 
Table 2 Efficacy of atezolizumab compared to docetaxel according to PD-L1 expression.

\begin{tabular}{lcccc}
\hline PD-L1 expression level & \% of patients & Hazard ratio for OS & P value & Hazard ratio for PFS \\
\hline Intent to treat & 100 & 0.73 & 0.0003 & 0.95 \\
IC O and TC 0 & 42 & 0.75 & 0.0215 & NR \\
IC $1 / 2 / 3$ or TC 1/2/3 & 57 & 0.74 & 0.0102 & NR \\
IC $2 / 3$ or TC $2 / 3$ & 30 & 0.67 & 0.0080 & NR \\
IC 3 or TC 3 & 17 & 0.41 & $<0.0001$ & 0.63 \\
\hline
\end{tabular}

OS, overall survival; PFS, progression-free survival; NR, not reported.
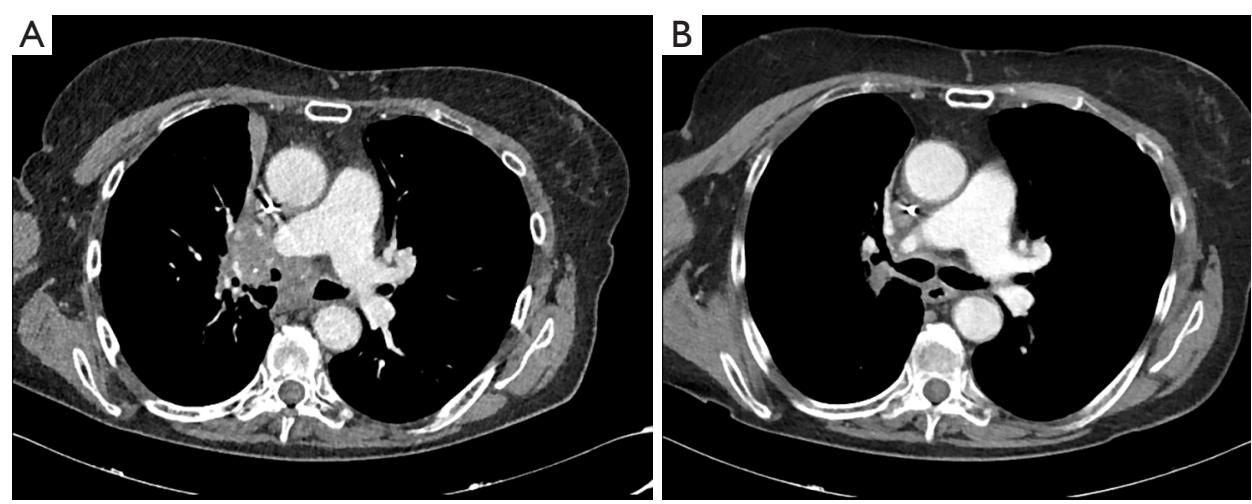

Figure 1 A 74-year-old female patient with locally advanced squamous cell carcinoma of the lung. (A) Progression after two regimens (chemoradiotherapy and chemotherapy) in May 2015; (B) ongoing response under durvalumab with re-opening of the right upper lobe (arrows), last updated in November 2017.

in favour of the Arm containing atezolizumab $(8.3 \mathrm{vs}$. 6.8 months; $\mathrm{HR}=0.62, \mathrm{P}<0.0001)$. In a pre-specified group with a T-effector gene signature, PFS was 11.3 vs. 6.8 months $(\mathrm{HR}=0.51, \mathrm{P}<0.0001)$, respectively. Severe adverse events were only slightly more in the four-drug combination ( $56 \%$ vs. $48 \%$ ) (10).

Now the overall survival data (the co-primary endpoint) as well as the survival results of the arm containing carboplatin, paclitaxel and atezolizumab are eagerly awaited.

\section{Durvalumab}

Durvalumab (MEDI 4736, Imfinzi $^{\circledR}$ ) is a human monoclonal IgG1 Antibody against PD-L1 without showing ADCC. In a phase $1 \mathrm{~b}$ Cohort, 228 patients with advanced NSCLC, of whom $56 \%$ received two or more prior lines of therapy, were treated with $10 \mathrm{mg} / \mathrm{kg}$ of body weight every 2 weeks. In 200 patients, response could be evaluated, showing a RR of $16 \%$ in the overall-heavily pretreated-NSCLCpopulation. In those showing PD-L1 expression, RR was
$27 \%$ compared to $5 \%$ in the PD-L1 negative group. Off note, PD-L1 negativity was defined as $<25 \%$ of tumor cells staining positive. About $66 \%$ of the responding patients had an ongoing response up to 54.4 weeks (Figure 1). Drug-related severe adverse events occurred in $8 \%$ of patients (11).

In the phase III PACIFIC trial, durvalumab was assessed as a consolidation therapy after definitive simultaneous chemoradiotherapy in stage III NSCLC. Seven hundred and thirteen patients were randomized and 709 received consolidation therapy by means of durvalumab or placebo in a 2:1 manner (473 and 236 patients, respectively). Coprimary endpoints were independently assessed PFS and OS. Randomisation was 1-42 days after chemoradiotherapy. The first endpoint is achieved and was published whereas the study remains blinded for OS assessment. PFS was clearly in favor for durvalumab with $16.8 \mathrm{vs}$. 5.6 months with placebo $(\mathrm{HR}=0.52 ; \mathrm{P}<0.001)$. The performance of the placebo arm is in line with previous publications taking into account, that randomization took place after 
Table 3 Hazard ratio (HR) for progression-free survival in the PACIFIC-trial in selected subgroups

\begin{tabular}{lll}
\hline Subgroup & HR & Confidence interval \\
\hline Smoking history & & \\
Smoker & 0.59 & $0.47-0.73$ \\
Nonsmoker & 0.29 & $0.15-0.57$ \\
Histology & & \\
Squamous & 0.68 & $0.50-0.92$ \\
Non-squamous & 0.45 & $0.33-0.59$ \\
Stage & & \\
IIla & 0.53 & $0.40-0.71$ \\
IIlb & 0.59 & $0.44-0.80$ \\
PD-L1 & & \\
$<25 \%$ & 0.59 & $0.43-0.82$ \\
$\geq 25 \%$ & 0.41 & $0.26-0.65$ \\
Unknown & 0.59 & $0.42-0.83$ \\
\hline
\end{tabular}

chemoradiotherapy in this trial (12). The RR was also higher with durvalumab (28.4\% vs. 16\%, $\mathrm{P}<0.001)$. Further results are shown in Table 3. Severe adverse events occurred in $29.9 \%$ under durvalumab and $26.1 \%$ under placebo. Pneumonitis is a great concern with immunotherapy after radiation. Treatment discontinuations because of this entity occurred in only $6.3 \%$ of patients under durvalumab and $4.3 \%$ under placebo (13).

Although these data are doubtless promising, some concerns remain: first, the protocol for chemoradiation was neither standardized nor part of the study protocol resulting in profound differences in the conduct of this procedure. Second, staging prior to treatment was not pre-specified. The only mandatory procedure was computed tomography of the chest resulting in imprecise anatomical staging. Third, we don't now, whether the PFS-benefit will result in a relevant OS-benefit or whether subsequent checkpoint inhibition in patients in the placebo arm will equalize OS.

Tremelimumab, a cytotoxic T-lymphocyte-associated protein 4 (CTLA-4) antibody was tested in a phase $1 \mathrm{~B}$-study in combination with durvalumab in NSCLC patients. One hundred and two patients received the combination showing that Durvalumab at $20 \mathrm{mg} / \mathrm{kg}$ every 4 weeks plus tremelimumab $3 \mathrm{mg} / \mathrm{kg}$ was the maximal tolerated dose with diarrhea, colitis and elevated lipase as the most frequent severe toxicities. Patients were mostly pretreated. RR was $23 \%$ with no clear correlation to the PD-L1 status (14). However, the subsequent phase III trial of first line durvalumab plus tremelimumab versus standardof-care platinum containing chemotherapy (MYSTIC, NCT02453282) was retracted from publication due to negative study results in a press release by the sponsor, Astra Zeneca. Whether both of the co-primary endpoints, PFS and OS or only PFS were not reached, is not known.

\section{Avelumab}

Avelumab (MSB0010718C, Bavencio $^{\circ}$ ) is a human IgG1 monoclonal antibody against PD-L 1 which has, in opposite to all other antibodies discussed above, ADCC properties. By this, besides of blocking PD-L1 an antibody dependent lysis of the target cell in the presence of natural killer cells and peripheral blood mononuclear cells (PMBC) takes place (15). This may enhance the cytotoxic effect of the compound in clinical settings. Avelumab has already gained accelerated approval for Merkel cell carcinoma and gastric cancer.

In a sub-cohort of the JAVELIN solid tumor $1 \mathrm{~b}$ trial, 184 patients with stage IIIB or IV lung cancer received $10 \mathrm{mg} / \mathrm{kg}$ avelumab every 2 weeks. The most concerning side effect was an infusion-related reaction occurring in $21 \%$ of patients ( $2 \%$ severe). In the group of patients receiving premedication (166 patients) the incidence of this adverse event was lower at all and as a severe adverse event (16\% and $1 \%$ respectively). Of note, this reaction mainly occurred after the first or second infusion of avelumab.

About $66 \%$ of patients had one and $33 \%$ of patients had $\geq 2$ prior lines of therapy. Twelve percent of patients achieved a response and $38 \%$ stable disease as best result. Median OS was 8.4 months. Notably the median in the duration of response was not reached at the time of the full publication (CI, 48.1 weeks - not reached) (16).

In the phase III Javelin lung 100 trial (NCT02576574) avelumab is tested against investigators' choice platinumbased chemotherapy as first-line treatment in advanced NSCLC. Coprimary endpoints are PFS and OS in the subgroup of patients with high (i.e., $\geq 25 \%$ of tumor cells) PD-L1 expression in the tumor, whereas those with moderate expression denote secondary endpoints. The trial is still recruiting and results are thus pending.

\section{Summary and conclusions}

With now three PD-L1 antibodies approved or in late 
clinical development for the treatment of NSCLC, the armament of clinically active checkpoint inhibitors is growing. Open issues are, whether there is a clinically relevant difference to $\mathrm{PD}-1$ antibodies or in-between the group of PD-L1 inhibitors. Especially the question whether ADCC enhances clinical activity or just toxicity, remains unanswered.

PD-L1 checkpoint inhibition with Atezolizumab is the first to show superior efficacy in combination with chemotherapy and bevacizumab in a phase III trial. According to a press release, the combination of the PDL1 inhibitor Durvalumab with the CTLA-4 antibody tremelimumab, however, seems not to be more effective than platinum based chemotherapy. The results of this trial are pending.

Another future direction will be the combination with other immunotherapeutics like Indoleamine-2,3dioxygenase (IDO) inhibitors. By reducing the availability of the essential amino acid tryptophane in the tumor environment, IDO prevents T-cell driven rejection and thus induces immune tolerance (17). IDO inhibitors like 1-methyl-D-tryptophan (NCT00739609) or indoximod (NCT02460367) are in early clinical development and are good candidates for combination with checkpoint inhibitors, however clinical data is missing.

Doubtless, the era of immune oncology as the "third wave" (18) of lung cancer treatment will continue to change the treatment of this devastating disease.

\section{Acknowledgements}

None.

\section{Footnote}

Conflicts of Interest: DF Heigener: Advisory Board: Boehringer Ingelheim, Roche, BMS, MSD, Pfizer, Lilly, Astra Zeneca, Chugai, Fresenius; Honoraria for Speeches: Boehringer Ingelheim, Roche, BMS, MSD, Pfizer, Lilly, Astra Zeneca; Travel Reimbursement: Boehringer Ingelheim, Roche, BMS, MSD, Pfizer, Lilly, AstraZeneca, Chugai, Fresenius. M Reck: Advisory Board: Boehringer Ingelheim, Roche, BMS, MSD, Pfizer, Lilly, Astra Zeneca, Chugai; Honoraria for Speeches: Boehringer Ingelheim, Roche, BMS, MSD, Pfizer, Lilly, AstraZeneca; Travel Reimbursement: Boehringer Ingelheim, Roche, BMS, MSD, Pfizer, Lilly, Astra Zeneca, Chugai, Fresenius.

\section{References}

1. Brahmer JR, Tykodi SS, Chow LQ, et al. Safety and activity of anti-PD-L1 antibody in patients with advanced cancer. N Engl J Med 2012;366:2455-65.

2. Topalian SL, Hodi FS, Brahmer JR, et al. Safety, activity, and immune correlates of anti-PD-1 antibody in cancer. $\mathrm{N}$ Engl J Med 2012;366:2443-54.

3. Ghiotto M, Gauthier L, Serriari N, et al. PD-L1 and PDL2 differ in their molecular mechanisms of interaction with PD-1. Int Immunol 2010;22:651-60.

4. Karunarathne DS, Horne-Debets JM, Huang JX, et al. Programmed Death-1 Ligand 2-Mediated Regulation of the PD-L1 to PD-1 Axis Is Essential for Establishing CD4(+) T Cell Immunity. Immunity 2016;45:333-45.

5. Akbari O, Stock P, Singh AK, et al. PD-L1 and PD-L2 modulate airway inflammation and iNKT-cell-dependent airway hyperreactivity in opposing directions. Mucosal Immunol 2010;3:81-91.

6. Pillai RN, Behera M, Owonikoko TK, et al. Comparison of the toxicity profile of PD-1 versus PD-L1 inhibitors in non-small cell lung cancer: A systematic analysis of the literature. Cancer 2018;124:271-7.

7. Butte MJ, Keir ME, Phamduy TB, et al. Programmed death-1 ligand 1 interacts specifically with the B7-1 costimulatory molecule to inhibit $\mathrm{T}$ cell responses. Immunity 2007;27:111-22.

8. Lee CK, Man J, Lord S, et al. Checkpoint Inhibitors in Metastatic EGFR-Mutated Non-Small Cell Lung Cancer-A Meta-Analysis. J Thorac Oncol 2017;12:403-7.

9. Rittmeyer A, Barlesi F, Waterkamp D, et al. Atezolizumab versus docetaxel in patients with previously treated non-small-cell lung cancer (OAK): a phase 3, openlabel, multicentre randomised controlled trial. Lancet 2017;389:255-65.

10. Reck M, Socinski M, Cappuzzo F, et al. Primary PFS and safety analyses of a randomized $\mathrm{Ph}$ III study of carboplatin (C) + paclitaxel (P) +/- bevacizumab (bev), with or without atezolizumab (atezo) in chemotherapy (chemo) naive, nonsquamous metastatic NSCLC (mNSCLC; IMpower 150). Ann Oncol 2017;28:abstr LBA1_PR.

11. Rizvi NA, Brahmer JR, Ou SH, et al. Safety and clinical activity of MEDI4736, an anti-programmed cell deathligand 1 (PD-L1) antibody, in patients with non-small cell lung cancer (NSCLC). J Clin Oncol 2015;33:8032.

12. Rizvi NA, Peters S. Immunotherapy for Unresectable Stage III Non-Small-Cell Lung Cancer. N Engl J Med 2017;377:1986-8. 
13. Antonia SJ, Villegas A, Daniel D, et al. Durvalumab after Chemoradiotherapy in Stage III Non-Small-Cell Lung Cancer. N Engl J Med 2015;373:123-35.

14. Antonia S, Goldberg SB, Balmanoukian A, et al. Safety and antitumour activity of durvalumab plus tremelimumab in non-small cell lung cancer: a multicentre, phase $1 \mathrm{~b}$ study. Lancet Oncol 2016;17:299-308.

15. Boyerinas B, Jochems C, Fantini M, et al. AntibodyDependent Cellular Cytotoxicity Activity of a Novel AntiPD-L1 Antibody Avelumab (MSB0010718C) on Human Tumor Cells. Cancer Immunol Res 2015;3:1148-57.

Cite this article as: Heigener DF, Reck M. Advanced non-small cell lung cancer: the role of PD-L1 inhibitors. J Thorac Dis 2018;10(Suppl 13):S1468-S1473. doi: 10.21037/ jtd.2018.01.112
16. Gulley JL, Rajan A, Spigel DR, et al. Avelumab for patients with previously treated metastatic or recurrent non-smallcell lung cancer (JAVELIN Solid Tumor): dose-expansion cohort of a multicentre, open-label, phase $1 \mathrm{~b}$ trial. Lancet Oncol 2017;18:599-610.

17. Moon YW, Hajjar J, Hwu P, et al. Targeting the indoleamine 2,3-dioxygenase pathway in cancer. J Immunother Cancer 2015;3:51.

18. Heigener DF, Reck M. Immunotherapy: the third wave in lung cancer treatment. Lancet Respir Med 2015;3:923-4. 